\title{
L'enseignement de la musique au lycée : mise en perspective de son évolution avec les enjeux globaux du système éducatif
}

\section{Eric Michon}

\section{(Q) OpenEdition}

\section{Journals}

Édition électronique

URL : http://journals.openedition.org/trema/364

DOI : $10.4000 /$ trema.364

ISSN : 2107-0997

Éditeur

Faculté d'Éducation de l'université de Montpellier

\section{Édition imprimée}

Date de publication : 1 décembre 2005

Pagination : 49-66

ISSN : $1167-315 X$

\section{Référence électronique}

Eric Michon, «L'enseignement de la musique au lycée : mise en perspective de son évolution avec les enjeux globaux du système éducatif », Tréma [En ligne], 25 | 2005, mis en ligne le 04 mars 2010, consulté le 19 avril 2019. URL : http://journals.openedition.org/trema/364 ; DOI : 10.4000/trema.364

Ce document a été généré automatiquement le 19 avril 2019

Trema 


\title{
L'enseignement de la musique au lycée : mise en perspective de son évolution avec les enjeux globaux du système éducatif
}

\author{
Eric Michon
}

Depuis la fin du XIXe siècle et la mise en place de notre système éducatif moderne, notre pays a toujours intégré l'enseignement de deux disciplines artistiques (la musique et le « dessin », initialement) dans le cursus scolaire des élèves. Plus récemment, la création d'un bac littéraire valorisant une dominante artistique («A6», musique et «A7», arts plastiques) a été instituée à la fin des années soixante. L'enseignement de la musique au lycée occupe toujours, néanmoins, une place à part dans le paysage éducatif français et ce, à triple titre :

- Obligatoire de la maternelle à la fin de la classe de troisième, cet enseignement artistique n'est plus qu'optionnel à partir de la seconde.

- La dénomination même de cette discipline artistique semble indiquer un changement de nature de l'enseignement dispensé : « éducation musicale » à l'école et au collège, elle devient « musique » au lycée. Il n'est pas anodin de faire remarquer que cette distinction trouve son pendant dans les intitulés des concours de recrutements d'enseignants du second degré : le CAPES d'éducation musicale et de chant choral destine plus particulièrement les professeurs à l'enseignement au collège, les titulaires de l'agrégation de musique ayant eux plutôt vocation à postuler dans les lycées. Nulle distinction de ce type n'existe dans la seconde discipline artistique présente dans notre système éducatif, les arts plastiques.

- Le recrutement des enseignants dans les options L-Arts - actuelle dénomination de la série obéit à une procédure spécifique, distincte du traditionnel mouvement national. L'institution considère en effet que des compétences particulières, de haut niveau, sont requises pour assurer cet enseignement qui bénéficie d'un horaire important - 3 heures en 
seconde, 5 heures en première et en terminale - et recrute ses enseignants en lycée dans le cadre d'un mouvement spécifique.

- Ces deux disciplines, « fondatrices » de la présence des arts au lycée, ne sont plus seules désormais : complétées depuis une quinzaine d'années par l'enseignement de l'histoire des arts, du théâtre, du cinéma-audiovisuel et plus récemment de la danse, elles participent à la définition d'une offre artistique enrichie et diversifiée. En marge de cette offre, une section plus professionnalisante est proposée à des élèves se destinant à un métier d'instrumentiste ou de danseur : la filière technologique Techniques de la Musique et de la Danse (TMD).

Notre propos se concentrera sur l'enseignement général au lycée, dans lequel

l'enseignement de la musique possède, à travers ses deux composantes - série L-Arts et option facultative - une finalité généraliste puisque ces deux offres ne sont pas conçues pour les seuls élèves inscrits dans l'enseignement spécialisé.

L'évolution de ces enseignements nous amènera dans un premier temps à étudier les contenus dispensés: une étude des différentes épreuves du baccalauréat proposées depuis 1970, une analyse des programmes limitatifs publiés chaque année pour fournir la matière à ces épreuves nous aidera ainsi à dégager la commande de l'institution en lien avec l'évolution des programmes et instructions officielles. Nous nous intéresserons ensuite de manière plus précise, à la lueur de ces textes proposés aux élèves aux épreuves du baccalauréat, aux nécessaires évolutions didactiques qu'elles ont progressivement induites : comment par exemple, à travers telle ou telle caractéristique d'une maquette d'épreuve, l'enseignement de la musique au lycée a notamment dû faire une plus large place à la pratique musicale des élèves, en plaçant notamment la voix comme vecteur premier de la transmission de connaissance. L'analyse de ces évolutions passées ou en cours, tant didactiques que concernant les contenus, nous permettra enfin de définir et de mesurer les enjeux de la présence de l'enseignement de la musique au lycée, dans toutes ses dimensions : dans le dispositif interne des enseignements artistiques, dans la cohérence de cette offre de la maternelle à l'Université ; elle permettra en outre de montrer à quel point ces évolutions de la discipline correspondent de manière beaucoup plus profonde à une conception globale de la pédagogie dans notre système éducatif.

\section{Evolution des contenus et commande institutionnelle}

\section{I.1 Bref panorama des épreuves de musique au baccalauréat}

Plutôt qu'une très traditionnelle entrée par les programmes et instructions officielles, nous avons en effet choisi dans un premier temps, pour analyser l'évolution des contenus de l'enseignement de la musique au lycée, de comparer différents textes d'épreuves proposés aux candidats entre 1970 et 2004: cette étude nous apparaît en effet particulièrement pertinente pour mesurer les changements intervenus en trente cinq ans et discerner ainsi l'évolution des compétences attendues des élèves dans cette même période.

Si l'épreuve de commentaire - première partie de l'épreuve écrite de la série littéraire option musique - est celle qui a subi le moins de changements depuis son apparition, de 
notables disparités sont toutefois à relever, qui dénotent à l'évidence des attentes fort différentes selon les époques. Voici trois textes ${ }^{1}$ - sessions de septembre des années 1970, 1971, 1972 - qui illustrent clairement l'esprit de l'épreuve à ses débuts ; que remarque-ton?

- L'étude de la structure de la pièce entendue est une constante - elle demeure aujourd'hui encore.

- La question finale, destinée à élargir le propos au-delà de l'analyse détaillée d'une œuvre précise et à solliciter les connaissances générales du candidat, reste un second invariant.

- En revanche, on remarquera qu'a cette époque la dernière question se centre soit sur la connaissance d'un compositeur ou d'une école (Quels sont les autres genres pianistiques illustrés par CHOPIN ?, 1971 ; L'intention descriptive qui domine l'ensemble de cette ceuvre est-elle une exception dans l'école russe au XIXe siècle ? Citez quelques exemples à l'appui de votre opinion), soit sur une forme musicale (Quelles différences constatez vous entre ce Prélude de WAGNER et les ouvertures de l'époque classique ?) - c'est d'ailleurs le cas général dans les années soixante-dix. L'étude demandée pour l'épreuve de juin 1976 de l'air Divinités du Styx de l'Alceste de GLÜCK se centre de la même manière sur la connaissance du compositeur (3e question, Citez d'autres opéras de GLÜCK) et d'un genre, l'opéra (4e et dernière question : Quels sont, aux XIXe et XXe siècles, les principaux représentants de cette forme musicale en France et en Allemagne? En vous appuyant sur un exemple précis, montrez l'influence d'un de ces musiciens sur l'évolution du théâtre lyrique). En 1979, ce sont les savoirs des candidats concernant la mise en musique du mythe de Faust qui sont interrogés, à partir de l'analyse d'un extrait de la Damnation de Faust.

5 Ces exemples démontrent, à cette époque, l'étendue des connaissances sollicitées - c'est notamment l'évolution historique de toutes les formes musicales qui doivent être connues, du Moyen-âge jusqu'à nos jours. Progressivement, le champ de cette dernière question du commentaire se réduit: En quoi cette œuvre (KindertodenLieder No 4, Gustav MAHLER) se distingue-t-elle ou se rapproche-t-elle d'un Lied ou d'une Mélodie que vous connaissez bien? - Juin 1983 ; Choisissez une œuvre vocale d'un autre compositeur. Commentezla brièvement et comparez-la à ce Lied (Des Lindenbaum, extrait du Winterreise de SCHUBERT) - Juin 1996, La Réunion.

6 Tout récemment enfin, la conclusion du travail demandé autour d'un extrait du Passio et mors domini nostri iusu christi secundum lucam de Kristof PENDERECKI réclamait de la même manière le recours à la comparaison avec une œuvre connue du candidat, cherchant en revanche - de manière très novatrice dans le contexte d'une question d'ouverture - à permettre un approfondissement de l'analyse de l'œuvre proposée.

Jusqu'à 1997, la seconde partie de cette épreuve écrite de la série obligatoire portait l'intitulé Analyse harmonique. Exercice académique s'il en est, les exigences requises révélaient systématiquement la mise en exergue de la tonalité générale du morceau, les principales modulations et emprunts, les principales cadences et l'analyse détaillée de plusieurs accords d'un texte spécifique issu du répertoire savant. A partir de la session 1998, l'épreuve devient "de technique musicale»; si l'analyse harmonique demeure, elle subit une évolution interne avec tout particulièrement une difficulté amoindrie du travail demandé aux élèves ${ }^{2}$ et se voit complétée par un exercice " $\mathrm{d}$ 'Invention de compléments musicaux $»^{3}$. Cette nouveauté a rapidement été abandonnée et, depuis deux ans (session 2003), la partie technique de l'épreuve s'est fondue dans celle du commentaire et vise à 
une analyse approfondie du langage musical employé dans un ou plusieurs extraits de la pièce étudiée.

8 Nous conclurons ce rapide panorama des épreuves de musique au baccalauréat avec quelques données concernant les oraux, qu'il s'agisse de ceux de l'épreuve facultative ou de la série littéraire. Remarquons tout d'abord que, jusqu'au milieu des années quatrevingt, un solfège chanté figure parmi les supports d'évaluation ${ }^{4}$. L'épreuve orale de commentaire de l'option obligatoire se fonde sur une œuvre inconnue des élèves jusqu'au début des années quatre-vingt dix. Dans tous les cas de figure, le candidat interprète vocalement ou avec son instrument de prédilection une œuvre qu'il a lui-même choisie et travaillée en dehors de ses cours au lycée. Jusqu'en 2002 enfin, l'épreuve facultative comprend une partie écrite dont la technicité évolue au fil des années : analyse d'accords (parfait Majeur ou mineur, septième de dominante ou "autre accord“), reconnaissance de timbres (deux instruments), dictée musicale puis simple dépistage de fautes ou partition à compléter, dictée rythmique qui disparait au début des années quatre-vingt dix. Depuis deux ans, la totalité de cette épreuve est orale.

\section{I.2. Programmes limitatifs}

9 Une autre dimension reste à prendre en considération dans ce bref descriptif, celle des programmes limitatifs que publie chaque année le Bulletin officiel, de leur existence ou non selon les options et du choix des œuvres qui y figurent.

L'épreuve facultative du baccalauréat a toujours intégré dans ses différentes composantes (histoire de la musique, solfège chanté, interprétation vocale ou instrumentale, exercices d'écoute) une partie orale centrée sur la connaissance et l'analyse d'une œuvre issue d'un programme obligatoire. Souvent valorisée par le barème de l'épreuve - un tiers des points au moins -, elle intégra plus récemment une connaissance du contexte esthétique et culturel de la pièce musicale. De six œuvres obligatoires initialement, le programme évolua progressivement vers trois - un moment complétées par trois autres pièces choisies par le professeur avec ses élèves - dont l'une relevait systématiquement de la période contemporaine. VARESE, BERIO, STRAVINSKY, MILHAUD, BARTOK... dans les années soixante-dix et quatre-vingt; LIGETI, APERGHIS, BOULEZ, DUTILLEUX, REICH, GRISEY... dans la décennie suivante. Une ouverture sensible se manifeste ces dernières années: si la connaissance de la diversité des écritures contemporaines - en plus du répertoire plus ancien, toujours d'actualité - reste incontournable ${ }^{5}$, une découverte par les élèves de terminale de pièces ne relevant pas de la musique savante occidentale est introduite: le jazz (quatre interprétations de Body and soul, 2001), les musiques traditionnelles extra-européennes (Musiques de Bali et Java, 2003-2004, tangos cette année) ; en attendant d'autres ouvertures : pourquoi pas la chanson française à texte ou les musiques amplifiées actuelles? 
11 Parallèlement, une évolution importante a été entreprise depuis trois ans avec la publication annuelle d'un programme limitatif correspondant aux quatre thématiques du programme de terminale de la série L-Arts option musique. Les mêmes volontés d'ouverture, de connaissance du patrimoine et des nouvelles écritures contemporaines transparaissent dans les choix proposés. Quelques exemples suffisent à illustrer cette politique de diversité, du Lamento d'Ariana de MONTEVERDI aux Folksongs de BERIO, du Dixit Dominus de HAENDEL à divers tangos issus des cultures populaires (GARDEL, PIAZZOLA dans une moindre mesure) et savante (STRAVINSKI, WEIL), des pièces pour piano préparé de CAGE à l'arrangement pour piano de la Chaconne de la Partita pour violon en ré mineur de BACH par Ferrucio BUSONI...

\section{I.3. Le sens de ces évolutions}

Que nous apprennent ces évolutions, de facto assez considérables, sur la légitime volonté de l'Institution d'impulser le changement pour adapter l'offre de formation musicale au lycée en fonction de ses propres exigences ? Les caractéristiques des différentes épreuves du baccalauréat, véritable définition des connaissances et compétences attendues des élèves au terme de leur scolarité obligatoire, ainsi que des divers programmes limitatifs indiquent de manière assez précise les orientations pédagogiques que fixe en termes de contenus d'enseignement l'Education nationale pour la formation musicale des élèves au lycée.

Elles sont de trois ordres :

- Passer d'une approche chronologique à une approche thématique des connaissances.

- Au début des années quatre-vingt dix a vu le jour, en effet une organisation des programmes de première et de terminale en douze thèmes d'étude qui, peu ou prou, respectaient l'évolution chronologique de l'histoire de la musique : des époques Renaissance et (surtout) Baroque à la fin du XIXe en classe de 1re, le XXe en Terminale. Cette conception ne faisait de facto que prolonger celle des précédents programmes : même traitement chronologique selon les niveaux - mais la classe de seconde n'était pas auparavant une classe « de détermination » et les époques les plus anciennes y étaient à l'étude -, avec une attention plus particulièrement portée tout au long de l'année de première sur les grandes formes musicales classico-romantiques. Depuis 1998, les instructions officielles ont déterminé, pour chaque niveau, une approche thématique de l'appropriation des connaissances en imposant quatre problématiques transversales par an ${ }^{6}$.

- D'une analyse musicologique traditionnelle, évoluer vers une connaissance plus globale des partitions.

14 L'analyse harmonique comme technique unique d'analyse du langage musical a vécu; la raison principale en est bien évidemment le caractère très restrictif de cette démarche, qui limite le travail sur partition aux œuvres tonalement analysables et rend ainsi impossible toute étude technique d'une écriture contemporaine. La nouvelle conception de l'épreuve écrite permet par ailleurs de renforcer la cohérence des apprentissages en 
resserrant dans le cours les liens entre pratiques musicales et connaissance des œuvres. Nous reviendrons sur cette question fondamentale un peu plus loin.

- Evoluer d'une centration sur les contenus académiques - analyse traditionnelle des musiques savantes occidentales, avec notamment hypertrophie des attentes sur les époques Baroque, Classique et Romantique - à une ouverture à toutes les musiques, savantes contemporaines - une réalité depuis plusieurs décennies déjà - mais également populaires de toutes origines.

La signification profonde de ces nouvelles orientations pédagogiques révèle ainsi une volonté de l'Institution de peser plus nettement sur la réalité concrète de cet enseignement, dans trois domaines notamment :

- Se démarquer de l'académisme de l'analyse musicologique traditionnelle. En clair, construire, après le collège - on pourrait également évoquer, dans une moindre mesure, l'école élémentaire - et en cohérence avec ce niveau d'enseignement, une véritable didactique de la discipline au lycée : élaborer un mode de transmission des connaissances adapté au public visé.

- Se singulariser, affirmer la spécificité de cette offre de formation à caractère éminemment généraliste en distinguant plus clairement son offre de formation de celle de l'enseignement spécialisé ; parallèlement (nous y reviendrons), le développement des partenariats avec des structures externes à l'Education nationale, dont les Ecoles nationales de musique et les Conservatoires, est encouragé.

- Adapter les contenus d'enseignement aux nouveaux profils d'élèves - issus de la démocratisation du lycée général, qui accueille désormais $60 \%$ environ d'une classe d'âge et à une demande forte de leur part qui doit être mise en relation avec l'importance de pratiques musicales très diverses chez les jeunes.

L'évolution des contenus d'enseignement de la musique au lycée révèle ainsi de manière prégnante un premier signe fort d'un pilotage renforcé de l'institution qui vise à donner une lisibilité et une cohérence accrue de son offre de formation musicale de la maternelle au baccalauréat. Voire plus encore : l'évolution récente du CAPES (épreuve sur dossier et, surtout, d'arrangement) en témoigne. L'étude qui suit des démarches mises en œuvre à ce niveau de la scolarité des élèves constitue un second élément renforçant cette analyse.

\section{Des démarches qui placent désormais la pratique au centre du processus d'apprentissage}

17 «Les pratiques musicales constituent le fondement de ce programme. Elles sont le moyen de l'acquisition des connaissances et des compétences; elles favorisent les échanges et s'enrichissent de la diversité des parcours des élèves. L'enseignement privilégie ainsi l'expression artistique individuelle et collective en s'appuyant prioritairement sur les pratiques vocales en sollicitant et renforçant ses compétences techniques. $»^{7}$

\section{II.1. Evolution de la didactique de la discipline}

18 Si les épreuves écrites et orales du baccalauréat définissaient notamment comme critères d'évaluation des élèves - outre la nécessaire maittrise de la technique du commentaire d'œuvre - la connaissance de l'histoire de la musique, la maîtrise de l'analyse harmonique traditionnelle et celle du déchiffrage chanté en clé de sol, les démarches mises en œuvre 
en classe devaient nécessairement correspondre à l'appropriation de ces contenus pour que les élèves puissent obtenir leur diplôme. De fait, ceux-ci y sont toujours parvenus les statistiques attestent de manière constante du succès de la formation - grâce notamment à leurs professeurs qui, d'une manière générale, maitrisaient remarquablement les savoirs à dispenser. D'une manière générale, le cours s'organisait en deux parties égales : histoire de la musique à partir de l'écoute et de l'analyse d'œuvres, et «travaux pratiques » qui permettaient à la fois la pratique du solfège chanté et l'analyse technique des accords et des modulations à partir de textes appropriés. Jusqu'au début des années quatre-vingt-dix, la pédagogie frontale, qui se fonde sur une démarche qui part des connaissances théoriques pour aboutir à leur illustration à partir d'exemples vécus par la pratique, est de mise, sans être contestée (ni contestable) par les instances pédagogiques. Il est vrai que l'objectif affiché de « $80 \%$ des élèves au niveau du baccalauréat » est encore récent et les effets induits par la démocratisation pas encore véritablement ressentis au lycée.

Les évolutions qui ont suivi ne sont pas seulement, loin s'en faut, la conséquence de l'afflux de lycéens consécutif à la mise en œuvre des objectifs de la loi d'orientation de 1989 ; elles sont aussi, comme nous l'avons déjà souligné, le signe d'une volonté de mise en cohérence des différents niveaux de l'offre de formation : école élémentaire, collège, lycée. Traduites de manière plus incisive dans les derniers programmes d'enseignements, elles induisent désormais une pédagogie inductive qui vise à placer l'élève en situation première d'activité musicale. De cette pratique naît l'appropriation de savoir-faire et de connaissances. Ainsi y est-il affirmé que les pratiques musicales "sont au centre de l'enseignement dispensé en classe. De l'interprétation d'un répertoire à l'étude ou à l'expérimentation du matériau musical, elles traversent les différents aspects du programme, soit comme moyens, soit comme objectifs."; partir du sensible pour aboutir à la connaissance théorique et culturelle, telle est désormais la démarche didactique préconisée. Il nous faut reconnaître toutefois que telle n'est pas toujours la réalité de terrain, tant la difficulté pour les professeurs reste grande d'opérer cette "mutation pédagogique" mais aussi de tenir compte d'une nouvelle réalité, celle d'une très forte hétérogénéité de leur public d'élèves désormais.

Corollaire de la construction - évoquée précédemment - de cette nouvelle didactique de la discipline au lycée, la cohérence visée entre les pratiques musicales et les thématiques du programme correspond trait pour trait avec les nouvelles maquettes d'épreuves du baccalauréat. Les différentes pratiques musicales s'appuient en effet sur des œuvres de référence (chantées, jouées, écoutées) qui recouvrent le plus souvent possible, à chaque niveau, les quatre thématiques. Comme au collège enfin, les facultés créatrices des élèves sont sollicitées, les pratiques proposées devant intégrer cette dimension essentielle de l'art musical: "Ces pratiques actives débouchent opportunément sur des démarches plus exploratoires sollicitant la créativité des élèves. Imaginer un nouveau phrasé, changer la modalité d'une mélodie, modifier une succession harmonique, monnayer des durées ou varier un thème sont 
alors quelques possibles dont la familiarité permet peu à peu de passer d'une attitude créative à une autre, plus ambitieuse et visant la création musicale».

Cette exigence plus fortement affirmée qu'auparavant d'une centration de l'enseignement de la musique au lycée sur la dimension concrète de cet art ne peut être remise en cause dans ses fondements théoriques : que serait en effet la musicologie... sans les œuvres, nées incontestablement d'une pratique? Les hommes ont-ils attendu l'écriture neumatique et l'imprimerie pour composer de la musique? Une question demeure néanmoins, celle de la réalisation et de la mise en œuvre de ces pratiques dans le cadre du cours.

\section{II.2. Les vecteurs d'une pratique musicale renouvelée} apprentissages admis - la contradiction est toujours possible -, la voix apparaît dès lors comme l'indispensable media de la construction du savoir: commun à tous, cet instrument se révèle également celui par qui passe l'émotion, dans le rapport ténu qu'il entretient avec le corps et avec l'individu. L'expérience que représente l'apprentissage polyphonique en classe d'un choral de Bach reste à n'en pas douter inoubliable pour qui la découvre, au lycée le plus souvent. Dès lors, pour l'enseignant, ce texte s'avère d'une richesse fantastique pour construire des exercices en tous genres, de nature harmonique bien sûr mais pas seulement: le rapport texte - musique, la notion de figuralisme, l'orchestration (le timbre), les dynamiques ... peuvent également faire l'objet d'une étude approfondie. De la même manière, ce que nous appelons communément " jeux vocaux ", qui débouchent naturellement sur des activités de création, peuvent conduire à l'écoute et l'étude du répertoire vocal contemporain.

Au-delà de la simple question didactique, le développement chez l'élève de solides compétences vocales participe à la construction de l'individu, la maîtrise de cet organe s'avérant d'une importance vitale dans l'équilibre personnel. Sans aller jusqu'à ces considérations extérieures - pourtant importantes -, la musique a de tous temps été portée par cet instrument si familier et pourtant si lointain qu'est la voix. Le moins que puisse réaliser un enseignement de la musique au lycée est bien la prise de conscience chez l'élève de ce qu'il recèle en lui.

- Le développement des nouvelles technologies éducatives 
Il s'agit là d'un axe de travail très différent, qui peut paraitre éloigné tant l'outil informatique est galvaudé - recelant parfois même un parfum d' "alibi pédagogique » dans notre système éducatif moderne. Une réalité s'impose toutefois : l'essentiel de la création musicale contemporaine, qu'il s'agisse de musique populaire ou savante, est désormais élaborée via ce support. Dans ses utilisations les plus poussées, il constitue même - plus qu'un outil - un des vecteurs de la création : la transformation en temps réel du son participe en effet à la réalité artistique de l'œuvre. Comment, ainsi, intégrer dans la transmission du savoir et dans le cadre de cette nouvelle didactique de l'enseignement de la discipline au lycée, cet outil que nous ne saurions qualifier de «nouveau » mais qui tarde à s'imposer dans les classes ${ }^{8}$ ?

- L'ordinateur est en premier lieu pour l'enseignant un outil facilitateur pour piloter le cours d'éducation musicale au lycée : découpe des plages de $\mathrm{CD}$ pour accéder directement à l'extrait musical choisi, réalisation de séquences pour accompagner dans le style telle ou telle interprétation d'élève, construction d'exercices, réalisation de documents multimédia pour permettre le travail en autonomie des élèves, exploitation de documents pédagogiques réalisés sous l'égide du Ministère.

- L'outil informatique permet surtout aux élèves, à partir de logiciels de traitement du son par exemple, de prendre conscience des vecteurs de la création musicale contemporaine (ou non) à partir d'une pratique. Il leur permet également d'accéder aux techniques rudimentaires (les «boucles») qui président à la confection des musiques de consommation courante. De la même manière et à partir d'une expérience vocale, les logiciels récents permettent de modifier la prestation d'un élève, d'en obtenir un rendu plus musical et de développer chez l'élève une auto-critique fondatrice d'un esprit analytique mieux fondé.

- La mise en situation d'activité créative de l'élève se révèlera grandement facilitée par l'utilisation de ces outils: séquenceurs, arrangeurs; l'éditeur de partition pourra développer des compétences accrues en lecture. Le développement de l'utilisation de ces logiciels permettra in fine d'évacuer de l'offre de formation technologique - TMD - le travail sur bande magnétique devenu obsolète mais qui continue d'être évalué au baccalauréat, malgré les efforts constants du Ministère de l'Education nationale pour faire évoluer cette malheureuse situation.

\section{- L'intégration des pratiques musicales personnelles des élèves}

A dessein, nous n'avons évoqué jusqu'ici de manière précise l'évolution récente des épreuves instrumentales des différentes options du baccalauréat général ; attardons-nous y désormais. Elles présentent toutes les deux une importante caractéristique commune. Qu'elles soient "articulées aux pratiques musicales menées en classe 》 - épreuve obligatoire de spécialité, série L - ou qu'elles "témoigne(nt) des pratiques musicales menées durant l'année scolaire » - épreuve facultative, toutes séries -, ces évaluations mettent clairement en exergue - même si les intitulés ouvrent la porte à une nécessaire souplesse - la nécessité pour les professeurs d'aider les élèves à se préparer en classe à leur prestation musicale à l'examen. Ces textes les incitent dès lors, en amont, à intégrer dans leur enseignement les pratiques musicales personnelles de leurs élèves. C'est probablement l'une des raisons qui a incité les concepteurs des programmes et de l'organisation des enseignements à attribuer 5 heures à l'option obligatoire. Les motifs de cette injonction 
récente doivent être recherchées dans les points soulevés plus haut : prise en compte de l'hétérogénéité nouvelle issue de l'afflux de jeunes qui, auparavant, n'accédaient pas au lycée, du caractère généraliste de cette offre de formation qui ne suppose pas de prérequis en enseignement spécialisé, d'une pratique musicale populaire très développée qui crée une demande forte - extrapolation de la notion de « validation des acquis »?

Cette demande de l'Institution reste déstabilisante pour des professeurs qui n'ont pas toujours l'habitude d'individualiser leur enseignement; des solutions existent pourtant, loin d'être si complexes que cela.

En voici quelques-unes :

- - Prise systématique d'information sur les pièces étudiées dans l'enseignement spécialisé ou dans le cadre d'une pratique personnelle - les effectifs par classe, très raisonnables, le permettent généralement.

- - Exploitation dans les cours de ces œuvres, en fonction des objectifs poursuivis : analyse, travail technique, écoute comparative, activité de création ...

- - Heures regroupant les différents niveaux et consacrées exclusivement à une pratique vocale et instrumentale collective ; exploitation des pièces étudiées à cette occasion dans les cours. Même principe avec l'œuvre chorale proposée dans le cadre de ce complément indispensable à l'enseignement obligatoire (ou facultatif).

- Les pratiques musicales collectives

Nous entrons là dans la problématique du projet, introduite dans le système éducatif au début des années quatre vingt. Toutes les études montrent que l'existence d'une pratique chorale exigeante facilite dans un lycée le rayonnement et la pérennité de la section musique. Pourquoi ? La raison en est très probablement que les élèves trouvent dans la réalisation de ce projet, et sa traduction concrète qu'est le concert de fin d'année, la finalisation de leur engagement dans l'orientation qu'ils ont choisie. De surcroît, ces adolescents sont le plus souvent rejoints par d'autres, extérieurs à l'option: la valorisation de leur choix s'en trouve renforcée. Plus simplement, le concert choral met l'accent sur la dimension artistique de l'enseignement de la musique et, de manière corollaire, sur la dimension du faire, de la pratique... qui est bel et bien le but ultime de cette formation artistique.

D'une étude chronologique à une approche thématique des contenus d'enseignements, d'une pédagogique frontale à une mise en situation d'activité musicale de l'élève : nous assistons aujourd'hui, au terme (?) d'une évolution qui dure depuis une vingtaine d'années, à l'émergence d'une didactique nouvelle - nous oserons dire plus simplement : d'une didactique - de la discipline au lycée qui se démarque d'un mode de transmission du savoir traditionnel et révolu. Légitime puisque cet enseignement est destiné à tous les élèves qui souhaitent une formation musicale généraliste et non aux seuls qui suivent déjà un cursus dans les structures spécialisées, cette évolution s'inscrit plus globalement dans une dynamique qui dépasse largement le cadre strict du développement au lycée de cette offre de formation artistique. 


\section{Les enjeux de l'enseignement de la musique au lycée}

\section{III.1. Mise en perspective avec une évolution globale de l'enseignement des disciplines}

Depuis le début des années quatre-vingt - et, peut-être même, depuis la dernière grande réforme du collège de 1975 -, l'Institution tente d'impulser de nouvelles modalités pédagogiques. Lentes à s'imposer, elles n'en transforment pas moins, progressivement mais avec toujours plus d'effectivité, la réalité quotidienne des enseignements. De quoi s'agit-il, plus précisément?

- En premier lieu, une demande toujours plus affirmée de pédagogie différenciée est réitérée de manière récurrente afin de répondre à l'hétérogénéité croissante des publics dans tous les cycles d'études. Les différentes réformes avancent toutes dans cette même direction qui demande aux enseignants de diversifier leurs méthodes de travail pour s'adapter aux différents profils d'élèves. L'intégration dans les cours de musique au lycée des pratiques instrumentales personnelles des élèves peut être perçue comme une déclinaison de cette première impulsion.

- La démarche de projet en est une seconde : après l'apparition des PAE (Projets d'action éducative) il y a plus de vingt ans, après l'élaboration et la mise en œuvre du projet d'établissement construit autour d'axes déclinés en actions, de nouveaux dispositifs ont vu le jour qui reposent sur cette même dynamique : parcours diversifiés, travaux croisés, travaux personnels encadrés, itinéraires de découverte... L'idée de différenciation des parcours n'est bien évidemment pas non plus étrangère à la promotion de ces dispositifs pédagogiques nouveaux dans notre système éducatif.

- De la même manière, le troisième axe sur lequel se développe l'impulsion du Ministère se situe en parfaite complémentarité avec les deux autres: la recherche d'une mise en cohérence des savoirs enseignés à travers la transdisciplinarité relève de cette même idée d'aide aux élèves en tentant de donner plus de sens aux enseignements.

De ces principes majeurs, hérités de recherches pédagogiques déjà anciennes, qui guident toutes les réformes ou tentatives d'évolutions du système éducatif de notre pays, résulte la mise en œuvre la plus concrète et sensible possible d'une pédagogie inductive qui vise à permettre l'appropriation par les élèves de savoirs, de savoir-faire et de notions à partir de leur propre activité. A l'évidence, un enseignement artistique comme la musique ne peut que trouver sa place dans ces modalités de transmission du savoir ; les thématiques des programmes participent de cette recherche de cohérence en permettant la transdisciplinarité - les rapports de la musique au texte, à l'image, à la société, à la danse, à la notion de temps... - tandis que la mise en œuvre de productions musicales donnant lieu aux concerts de fin d'année s'inscrit très clairement dans cette dynamique de projets que l'Institution appelle de ses vœux. De la même manière, le positionnement central des pratiques, moyens d'appropriation des notions et des concepts, contribue à donner du sens aux apprentissages. 

options. Peu de lycées proposent en effet un cursus complet d'enseignement facultatifs ceux-ci sont trop souvent réduits à une formation d'une ou deux heures par semaine, en terminale exclusivement -, tandis que les effectifs sont très variables d'un lycée à un autre. Concernant les séries L-arts, le moins que l'on puisse dire est qu'elles ne souffrent pas d'une pléthore de candidats. Quelques chiffres illustreront notre propos :

- Seuls 1100 élèves se présentent aux épreuves du bac L-Arts Musique (plus de 6000 en Arts Plastiques)

- Bien meilleure concernant l'option facultative (12500 candidats, 15500 en Arts plastiques), la situation est en trompe l'œil : un tiers seulement suivent réellement les cours au lycée, les autres se présentent en candidats libres. 
Les causes de cette faiblesse quantitative sont connues : réputation de grande technicité de notre discipline - il est «impossible de suivre cet enseignement si l'on ne sait pas lire la musique et si l'on ne sait pas jouer d'un instrument... »-; difficulté croissante pour les chefs d'établissement d'établir des emplois du temps permettant à tous les élèves désireux de s'inscrire à l'option facultative et de la suivre effectivement. complète: s'il n'est pas discutable que la pratique musicale nécessite un véritable investissement, la primauté donnée au travail vocal peut pallier la pratique régulière d'un instrument. Les contenus d'enseignements de la classe de seconde sont enfin conçus pour pouvoir être suivis par tout élève de fin de troisième autorisé à passer en seconde et ayant des résultats satisfaisants en éducation musicale.

- Le positionnement de l'option obligatoire dans la série littéraire.

Un dernier chiffre éclairera la difficulté réelle dans laquelle se trouvent nombre de séries L-Arts Musique : parmi les élèves qui se présentent à l'option facultative, près de $75 \%$ suivent une filière scientifique ou économique, $50 \%$ sont en terminale $\mathrm{S}$. C'est donc bien le positionnement de l'option lourde dans un cursus littéraire qui pose problème, et ce à la différence des autres termes de l'offre d'enseignement artistique au lycée: les plasticiens, historiens des arts, ceux qui suivent les options cinéma ou théâtre possèdent pour la plupart, en effet, des profils à dominante littéraire. Le paradoxe est ainsi bien réel : l'art musical est celui qui attire le plus de jeunes mais sa présence dans le cursus optionnel au lycée est fragile. L'enseignement musical au lycée s'avère pourtant plus que jamais nécessaire.

- Un rôle d'interface entre l'enseignement obligatoire et l'Université.

Autre enjeu d'importance en effet, celui de la continuité et de la cohérence du dispositif institutionnel: si, comme nous avons tenté de le montrer, celle-ci est en passe d'être atteinte au plan didactique, il reste désormais à assurer la pérennité de l'enseignement de la discipline de la maternelle à l'Université. Son caractère optionnel au lycée confère à ce niveau d'étude un statut de charnière, palier décisif dont les professeurs - ceux exerçant au collège ayant en charge les élèves de troisième comme ceux en poste au lycée - doivent prendre conscience. Dispenser au lycée un enseignement accessible à tous, fondé sur une pratique artistique à la fois attractive et exigeante et qui intègre par ailleurs les nouvelles techniques de traitement du son, c'est d'une part générer une information plus aisée en amont puisque la cohérence avec ce qui est proposé au collège s'en voit renforcée ; c'est d'autre part motiver plus d'élèves pour les études universitaires dans cette branche et alimenter les deux voies de l'enseignement supérieur : la recherche musicologique, mais aussi la préparation des concours de recrutement d'enseignants, autre enjeu fort de la discipline puisque notre système éducatif doit beaucoup recruter dans les prochaines années. Nous ferons à nouveau remarquer à ce sujet l'évolution sensible du CAPES qui induit par exemple dans les schémas de formation universitaires des ouvertures accrues à toutes les musiques, mais aussi aux nouveaux outils technologiques et informatiques. 


\section{III.3. L'ouverture vers les partenaires extérieurs}

textes, instructions officielles ou préconisations pédagogiques, a donc globalement permis de réussir la nécessaire mise en cohérence des démarches et des contenus dispensés avec l'ensemble du dispositif éducatif issu des récentes réformes. Et ce mieux peut-être - plus rapidement surtout - que d'autres disciplines. La fragilité du positionnement demeure pourtant, faute probablement que soient encore réunies toutes les conditions de la réussite de cet enseignement :

- l'existence d'une pratique vocale collective qui propose chaque année un "grand" projet, exigeant (musique savante le plus souvent), faisant intervenir un partenaire musical extérieur ;

- la présence, de manière complémentaire à l'enseignement L-Arts Musique, d'une option facultative se révèle un facteur dynamisant très fort pour la vie de la musique dans l'établissement (chorale et pratiques musicales plus développées) ;

- l'ouverture sur le monde artistique local, la mise en œuvre de partenariats ;

- l'ouverture culturelle à toutes les musiques.

Faute, également, que le dispositif institutionnel s'adapte à la réalité sociologique des élèves motivés par ce type d'enseignement. Il paraît ainsi urgent, voire vital, que l'offre de formation musicale au lycée soit ouverte à un nombre accru d'élèves, issus de toutes séries 9 . Dans un contexte de réduction des moyens, une carte des options artistiques, qui permettrait une véritable mutualisation des moyens entre les lycées d'un même bassin, 
pourra-t-elle voir le jour? Autant de questions qui décideront de l'avenir de l'enseignement de la musique au lycée...

\section{ANNEXES}

Proposition d'organisation d'une offre de formation « musique » commune à toutes les séries :

\begin{tabular}{|l|l|l|}
\hline Seconde & Première & Terminale \\
\hline $\begin{array}{l}\text { Tronc commun facultatif / } \\
\text { obligatoire }\end{array}$ & $\begin{array}{l}\text { Enseignement de spécialité } \\
(3 \mathrm{~h})\end{array}$ & $\begin{array}{l}\text { Enseignement de spécialité } \\
(3 \mathrm{~h})\end{array}$ \\
\hline $\begin{array}{l}\text { Tronc commun facultatif / } \\
\text { obligatoire }\end{array}$ & $\begin{array}{l}\text { Tronc commun facultatif / } \\
\text { obligatoire }\end{array}$ & $\begin{array}{l}\text { Tronc commun facultatif / } \\
\text { obligatoire }\end{array}$ \\
\hline CHORALE (2h) - obligatoire (ouvert à tous les élèves de manières facultative) \\
\hline PRATIQUES MUSICALES COLLECTIVES (1) - obligatoire \\
\hline
\end{tabular}

Horaire de base du professeur : 16h ; sur les mêmes critères - enseignement réglementaire assuré pour les options obligatoire et facultative, avec chorale : $21 \mathrm{~h}$

\section{Annexe 1 : textes d'épreuves de commentaires, série A6 (septembre $1970,1971,1972)$}

1970

A. Commentaire d'une œuvre musicale

Richard WAGNER, prélude de Lohengrin, (édition Durand, partition d'orchestre, in-16).

1/ Préciser la structure de ce Prélude.

2/ Comment, par l'orchestration, WAGNER vous semble-t-il traduire l'esprit supraterrestre de la légende?

3/ Citez d'autres œuvres dramatiques de WAGNER commençant par un Prélude.

4/ Quelles différences constatez-vous entre ce Prélude de WAGNER et les ouvertures de l'époque classique?

1971

A. Commentaire d'une œuvre musicale CHOPIN, 15e Prélude (piano) édition Durand.

1/ Donner le plan de ce Prélude.

2/ En quoi ce Prélude est-il romantique? 
3/ Quels sont les éléments de cette œuvre qui vous paraisse les plus saisissants?

4/ Quels sont les autres genres pianistiques illustrés par CHOPIN?

1972

MOUSSORGSKY, Gnomus (extrait des tableaux d'une Exposition, $\mathrm{n}^{\circ} 1$ ).

Orchestration de Maurice RAVEL (édition Doosey et Hawkes, partition d'orchestre in-16, p. 6 à 20).

1/ Préciser le plan général de ce morceau.

2/ Montrer par quels moyens musicaux MOUSSORGSKY a su évoquer ce personnage étrange?

3/ Pouvez-vous expliquer en quoi les instruments choisis par Maurice Ravel contribuent à enrichir la description du personnage?

4/ L'intention descriptive qui domine l'ensemble de cette œuvre est-elle une exception dans l'école russe au XIXe siècle ? Citez quelques exemples à l'appui de votre opinion.

\section{Annexe $2:$ L"Invention de compléments musicaux"}

Epreuve de technique musicale du baccalauréat L-Art, juin 1998 :

à partir du texte musical ci-dessous (extrait) :

W.A.MOZART, Rondo, extrait de la Sonatine viennoise $\mathrm{n}^{\circ} 1 \mathrm{en}$ Do majeur.

Allegro Tonalité :

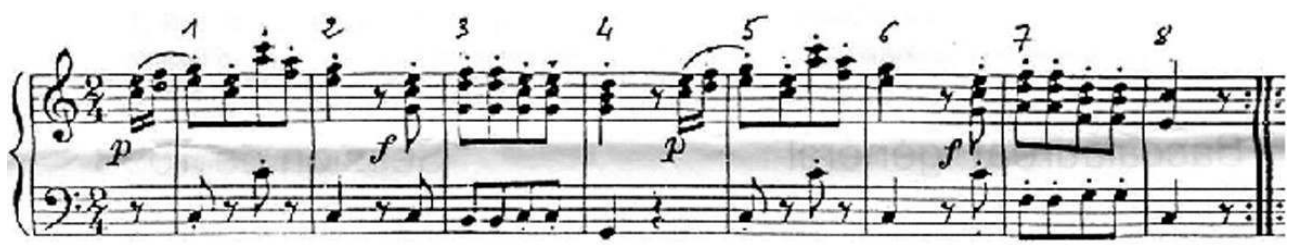

L'exercice suivant est demandé :

Yuvention de compléments nutusicaux ( sur 4)

En vous inspirant des dessins rythmiques proposes et en respeclant dans ses grandes lignes le schéma harmonique du textc de Mozart, écrivez une variation de la ligne mélodique supéricurc, du 2 ème tenips de la mesure 8 (dernière croche) à la fin.

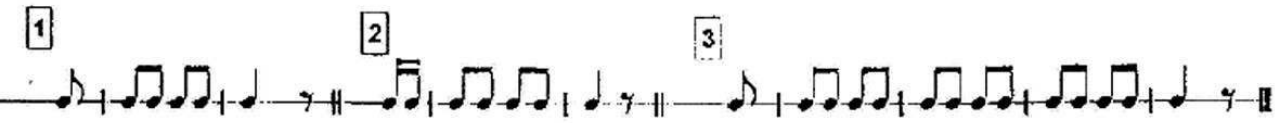

\section{Annexe 3 : Solfèges chantés et épreuves facultative}

Epreuve de technique musicale du baccalauréat L-Art, juin 1998 : 
a/ Série A3, juin 1987 (extrait)

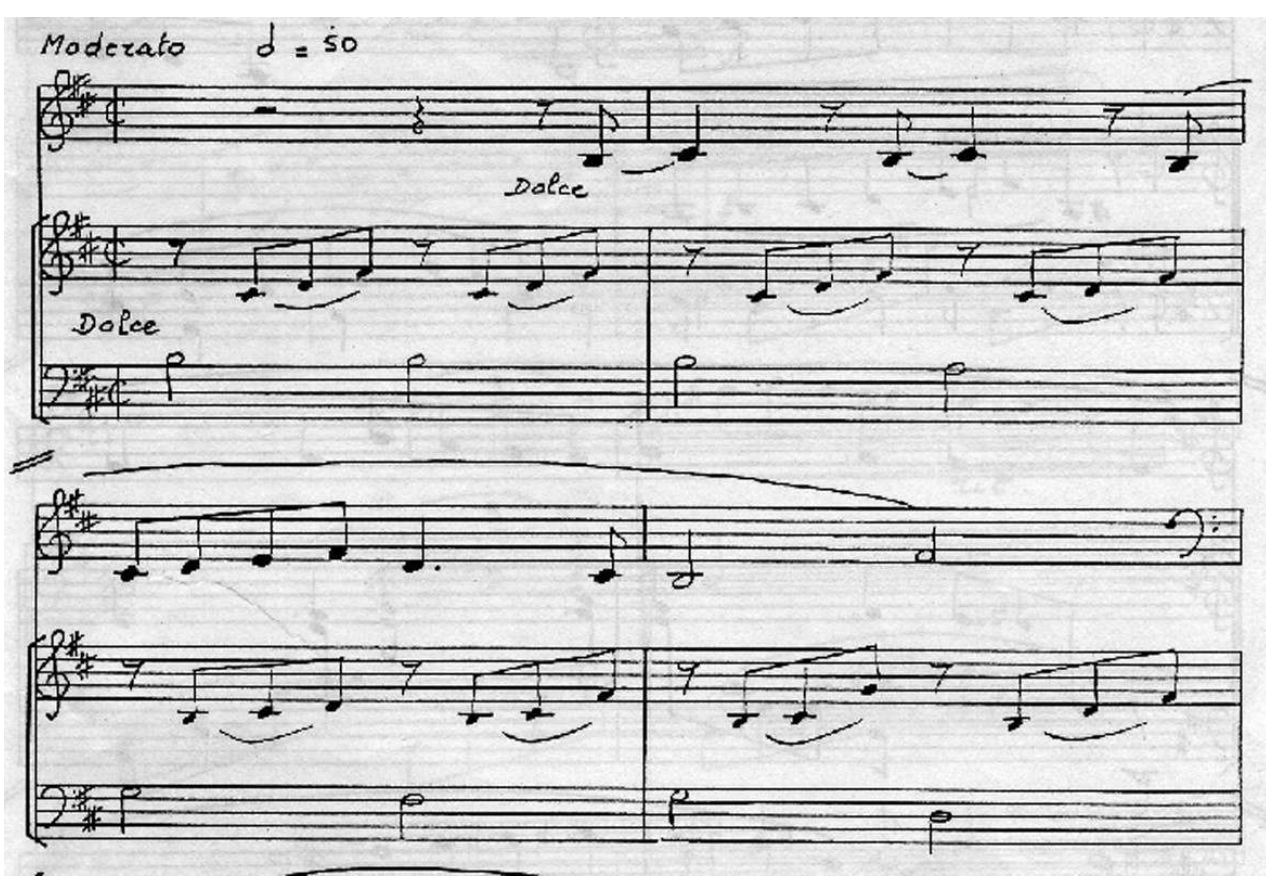

b/ épreuve facultative, Paris, 1987 (extrait)

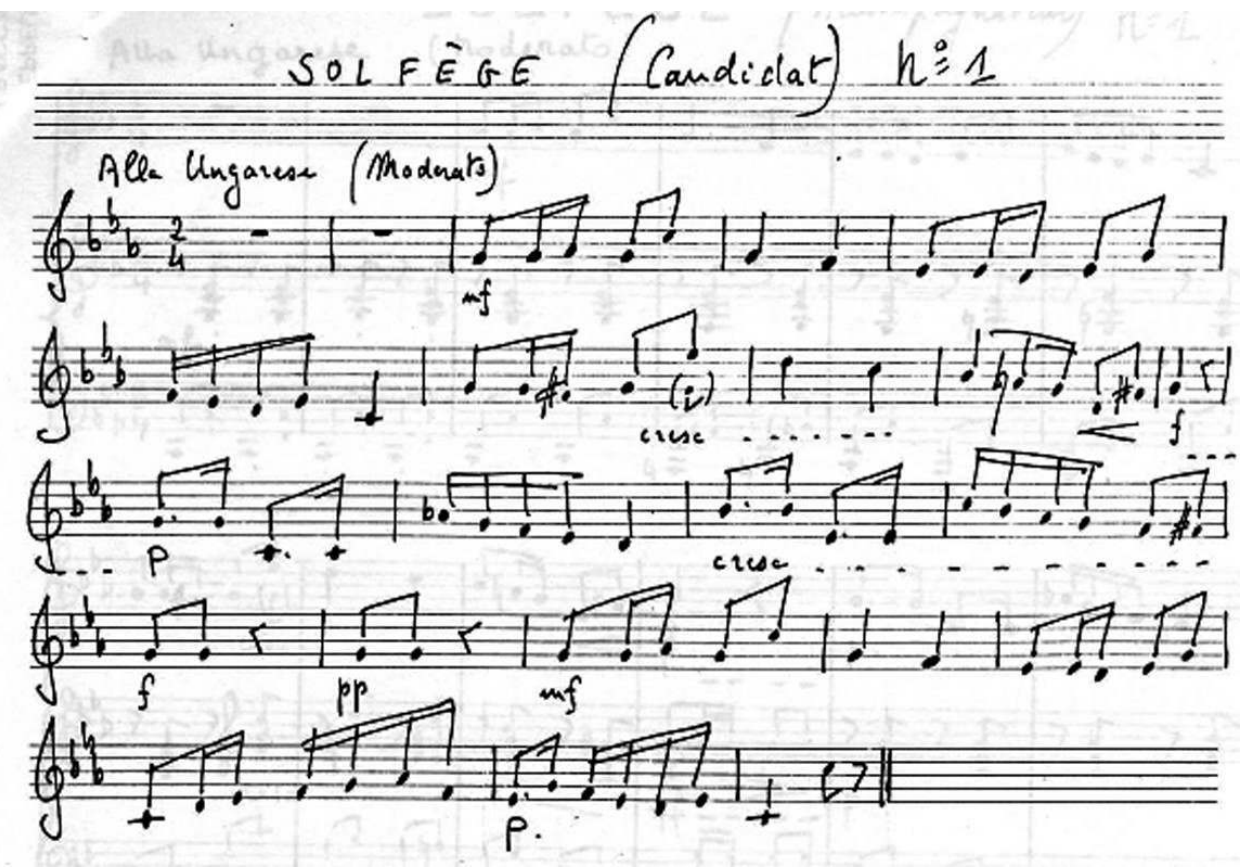




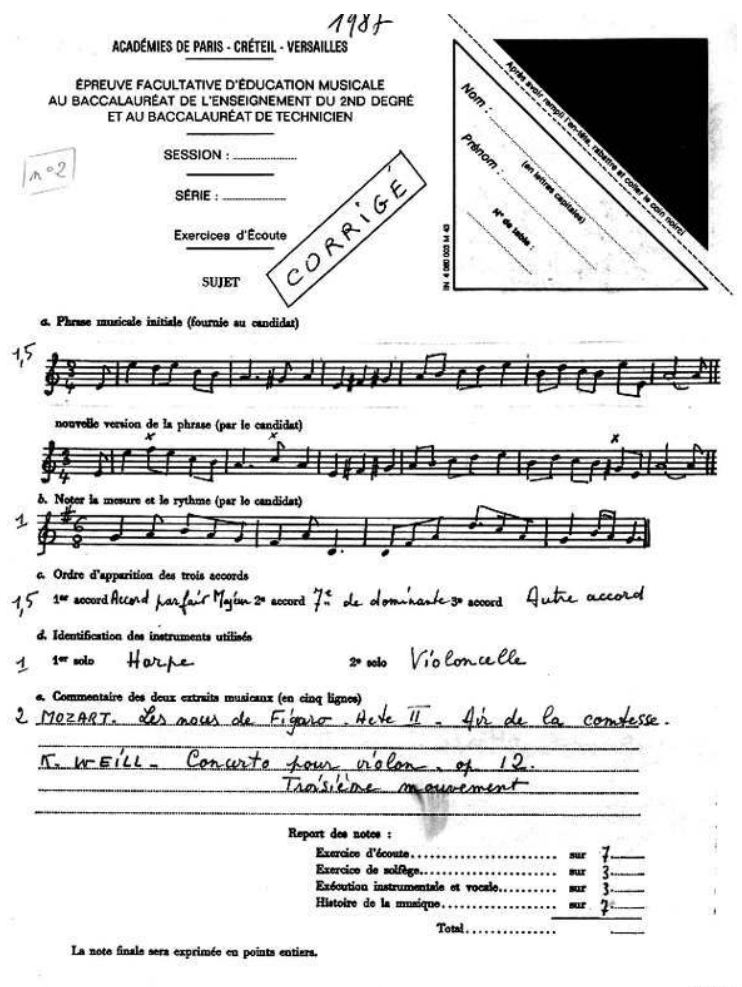

\section{NOTES}

1. Les œuvres étudiées sont respectivement : Prélude de Lohengrin (WAGNER), 15e prélude pour piano de CHOPIN, Gnomus, extrait des Tableaux d'une exposition (MOUSSOGSKI-RAVEL). L'intégralité de ces textes se trouve en annexe 1.

2. L'accord le plus complexe à analyser était - de même que dans les sessions suivantes - le 1er renversement d'une septième de dominante, difficulté à mettre en parallèle avec l'analyse d'accords de septième diminuée (très fréquente dans les années 70) ou, plus rarement de neuvième de dominante - Mazurka de CHOPIN, Juin 1979.

3. cf. annexe 2 .

4. Voir annexe 3 : extrait d'un texte de l'épreuve du baccalauréat A6 de juin 1979, de juin 1987 en série A3, de l'épreuve facultative de 1987 enfin.

5. Sud de Jean-Claude RISSET (2002), Songbooks de John CAGE (2003), Miserere et Cantus in memory of Benjamin BRITTEN D'ARVO PÄRT (2003-2005).

6. En seconde : les rapports de la musique au texte, les rapports de la musique à la société, les rapports du son et de l'image, les métissages musicaux. En première: Musique et expression du Sacré, Musique et danse, Musique et recherches formelles, Musique et drame. En terminale : Voix, texte et musique, Musique populaire-musique savante, Musique et timbre, Musique et Temps.

7. Programme actuel de la classe de Première.

8. Nous ne retiendrons pas ici l'argument spécieux du "manque de moyens" : la plupart des salles de classe en lycée sont équipées d'appareils multimédia performants, souvent à la hauteur de l'intérêt que leur portent les enseignants.

9. Nous émettons en annexe une proposition en ce sens. 


\section{RÉSUMÉS}

L'enseignement des arts au lycée, s'il ne se limite plus dorénavant seulement à l'éducation musicale et aux arts plastiques, n'en accorde pas moins une place prépondérante à ces deux disciplines. Eric Michon analyse les textes officiels et les contraintes liées à un enseignement optionnel. Il compare enfin les changements successifs relatifs aux épreuves du baccalauréat.

Even though teaching Arts in secondary schools is not any more limited to music teaching and art teaching, these two subjects are nevertheless given a major importance. Eric Michon examines official documents and the restraints imposed by teaching a non compulsory subject. He eventually draws a comparison between the various changes undergone by the tests at the "baccalauréat" examination.

\section{AUTEUR}

\section{ERIC MICHON}

IA-IPR Orléans-Tours, Antilles-Guyane 